\title{
Erratum to: Discovery of new Mycobacterium tuberculosis proteasome inhibitors using a knowledge-based computational screening approach
}

\author{
Rukmankesh Mehra $^{1}$ - Reena Chib ${ }^{2,4}$. Gurunadham Munagala ${ }^{3,4}$. \\ Kushalava Reddy Yempalla ${ }^{3,4}$. Inshad Ali Khan ${ }^{2,4}$ - Parvinder Pal Singh ${ }^{3,4}$. \\ Farrah Gul Khan ${ }^{2}$ - Amit Nargotra ${ }^{1,4}$
}

Published online: 26 October 2015

(C) Springer International Publishing Switzerland 2015

\section{Erratum to: Mol Divers (2015) 19:1003-1019 \\ DOI 10.1007/s11030-015-9624-0}

In the original publication of the article, the first sentence of abstract contained a mistake. The correct sentence should read as "Mycobacterium tuberculosis bacteria cause deadly infections in patients".

The online version of the original article can be found under doi:10.1007/s11030-015-9624-0.

Amit Nargotra

amitnargotra@yahoo.com; anargotra@iiim.ac.in

Farrah Gul Khan

fairygk@rediffmail.com

1 Discovery Informatics Division, CSIR-Indian Institute of Integrative Medicine, Canal Road, Jammu 180001, India

2 Clinical Microbiology Division, CSIR-Indian Institute of Integrative Medicine, Canal Road, Jammu 180001, India

3 Medicinal Chemistry Division, CSIR-Indian Institute of Integrative Medicine, Canal Road, Jammu 180001, India

4 Academy of Scientific and Innovative Research, CSIR-Indian Institute of Integrative Medicine, Canal Road,

Jammu 180001, India 